\title{
FLOWS ON FIBRE BUNDLES
}

\author{
BY
}

J. L. NOAKES

Abstract. Conditions are given under which a fibrewise flow on a fibre bundle must have a nonempty catastrophe space.

1. The problem. When we formulate the catastrophe theory of $R$. Thom globally we have a fibre bundle $E$ over a connected finite CW-complex $B$. The fibre $M$ of $E$ is a closed $C^{r}$ manifold, and the structure group of $E$ is a subgroup of the group Diff $M$ of $C^{r}$ diffeomorphisms $M \rightarrow M$ with the $C^{r}$ topology $(r>1)$. We say that $E$ is a $C^{r}$ bundle for short. Then $B$ is the space of observables and $M$ is the manifold of internal variables. Let Vect $M$ be the space of $C^{r-1}$ vector fields on $M$ with the $C^{r-1}$ topology. We define an action ' ' of Diff $M$ on Vect $M$ by means of the identity $f \cdot V=(d f) V f^{-1}$ where $f \in \operatorname{Diff} M, V \in$ Vect $M$. In catastrophe theory the bundle with fibre Vect $M$ associated with $E$ has a cross-section. We think of this cross-section as a family $V_{b}(b \in B)$ of fibrewise $C^{r-1}$ vector fields on $E$.

We next define an attractor of a vector field. The definition in $[5, \S 4.1]$ seems to be imprecise and we use the following definition instead.

Definition. An attractor of $V \in$ Vect $M$ is a closed invariant subspace $A$ of $M$ such that

(i) there is an invariant neighbourhood $U$ of $A$ for which $\cap_{t>0} \phi_{V t} U=A$,

(ii) some trajectory of $V$ is dense on $A$ (here $\phi_{V}$ is the flow on $M$ corresponding to $V$ ).

Perhaps an attractor ought to satisfy additional conditions but these would not affect our main results.

We suppose that we are given a convention, namely an assignment to each $b \in B$ of an attractor $A_{b}$ of $V_{b}$. We think of a convention as a physical law, and of $A_{b}$ as the physical state of $B$. Then $b \in B$ is said to be regular when it has a neighbourhood $W$ for which there is a fibre-preserving homeomorphism $h: E \mid W \rightarrow W \times E_{b}$ onto the trivial bundle satisfying

(i) $h \mid E_{b}$ is the identity,

(ii) $h \mid A_{c}=A_{b}$ and $h \phi_{V_{c}}=\phi_{V_{b}} h$ for all $c \in W$.

The points that are not regular make up the catastrophe subspace $K$ of $B$.

The purpose of this paper is to study the following problem. Suppose that we are given $E$ and a closed connected subspace $F$ of $M$. Then we wish to decide whether $K$ can be empty with $A_{0}=F$, that is to say whether there is a family $V_{b}(b \in B)$ of fibrewise $C^{r-1}$ vector fields on $E$ and a convention such that $K$ is empty and $A_{0}=F$. Here 0 is the basepoint of $B$.

Received by the editors January 16, 1979 and, in revised form, June 5, 1979.

1980 Mathematics Subject Classification. Primary 55R10; Secondary 58F25.

(c) 1980 American Mathematical Society 0002-9947/80/0000-0269/\$02.75 
If $K$ can be empty with $A_{0}$ a point then the assignment $b \mapsto A_{b}$ defines a cross-section of $E$. Conversely let $s$ be a cross-section of $E$. Then $K$ can be empty with $A_{0}$ a point. To prove this we argue as follows. The tangent bundles $T E_{b}$ of the manifolds $E_{b}(b \in B)$ make up a vector bundle $T F E$ over $E$. Choose a Riemannian metric on $T F E$ and let $T$ be $T F E \mid s(B)$. Then $T$ is a Riemannian vector bundle over $B$ and the $T_{b}$ are the tangent spaces at $s(b)$ to the manifolds $E_{b}(b \in B)$. Using the compactness of $B$ we choose $\delta>0$ so that the fibrewise exponential map $\varepsilon: T \rightarrow E$ maps the open disc bundle $B_{\delta}$ of radius $\delta$ homeomorphically into $E$. Then $N=\varepsilon\left(B_{\delta}\right)$ is a neighbourhood of $s(B)$ in $E$. We identify $T$ with its own fibrewise tangent bundle and for $e \in E_{b}$ we define $V_{b}(e)$ to be either 0 or

$$
\left(-d \varepsilon_{b}(t)\right) \exp (-\sec \theta)
$$

accordingly as $e \notin N$ or $e=\varepsilon(t)$ for $t \in B_{\delta}$. Here $\theta=\pi\|t\| / 2 \delta$. Taking $A_{b}=$ $\{s(b)\}$ we see that $K$ is empty.

In general when $K$ is empty the $A_{b}$ make up a fibre bundle $A$ over $B$. In some cases $A$ is a $C^{r}$ bundle and there is a fibrewise $C^{r}$ embedding $f: A \rightarrow E$. By this we mean that $f$ is a fibre-preserving map such that

(i) $f_{b}: A_{b} \rightarrow E_{b}$ is a $C^{r}$ embedding.

(ii) $f_{b}$ varies continuously in the $C^{r}$ topology with $b \in B$.

In $\$ \S 2$ and 3 we prove conditions necessary for the existence of fibrewise $C^{r}$ embeddings, and in $\$ 4$ we apply these results to our original problem. Our main results assert that under certain conditions the catastrophe space $K$ must be nonempty.

2. A related problem. Let $E, A$ be $C^{r}$ bundles with fibres closed $C^{r}$ manifolds $M, F$ over a connected finite $\mathrm{CW}$-complex $B(r \geqslant 1)$.

Lemma 1. Let $f: A \rightarrow E$ be a fibrewise $C^{r}$ embedding. Then the complement $E-f(A)$ is a $C^{r}$ bundle over $B$.

This requires only a local proof, and so we suppose that $E=B \times M$ and that there is a $C^{r}$ trivialization $g: B \times F \rightarrow A$. Then it suffices to extend $f g$ for each $b \in B$ over some neighbourhood $U$ of $b$ to a $C^{r}$ trivialization $h: U \times M \rightarrow E \mid U=$ $U \times M$. But this can be done because of the result, due to R. S. Palais, that the evaluation map on spaces of $C^{r}$ embeddings is locally trivial. We refer to [2] for a short proof of Palais' theorem.

Two fibrewise $C^{r}$ embeddings $f_{0}, f_{1}: A \rightarrow E$ are said to be isotopic when there is a fibrewise $C^{r}$ embedding $F: A \times[0,1] \rightarrow E \times[0,1]$ over $B \times[0,1]$ such that

$$
F\left|A \times\{0\}=f_{0}, \quad F\right| A \times\{1\}=f_{1} .
$$

Applying Lemma 1 to $F$ and using $[3,11.4]$ we have the following lemma.

Lemma 2. Let $f_{0}, f_{1}: A \rightarrow E$ be isotopic fibrewise $C^{r}$ embeddings. Then the $C^{r}$ bundles $E-f_{0}(A), E-f_{1}(A)$ are equivalent.

Let $E, A$ be orthogonal sphere bundles of fibre dimensions $q>p>1$ and let $f$ : $A \rightarrow E$ be a fibrewise $C^{r}$ embedding. We suppose that $f$ is orthogonal when 
restricted to the fibre over the basepoint 0 so that, in particular, the $f_{b}: A_{b} \rightarrow E_{b}$ are unknotted when $q=p+2$.

Problem. (i) Is there a fibrewise orthogonal embedding of $A$ in $E$ ?

(ii) If so then is $f$ isotopic to a fibrewise orthogonal embedding?

An affirmative answer to (i) would mean that $E$ was the fibre join of $A$ with an orthogonal $q-p-1$-sphere bundle. The Whitney duality theorem would then give conditions on the Stiefel-Whitney classes of $E$ necessary for the existence of $f$.

REMARK. If $A$ has a cross-section $s$ (for example if $A$ is an oriented circle bundle and $\left.H^{2}(B ; Z)=0\right)$ then the $d f\left(T F A_{s(b)}\right)$ define an orthogonal subbundle of $E$. We can identify this subbundle with $A$ and so there is a fibrewise orthogonal embedding of $A$ in $E$.

When $A$ does not have a cross-section we can still obtain a condition necessary for the existence of $f$ by pulling everything back over the principal bundle associated with $A$. We can then apply our remark, together with the Whitney duality theorem. However we shall do better than this.

Let $W$ be the path component of the orthogonal embeddings in the space of $C^{r}$ embeddings of $S^{p}$ in $S^{q}$ with the $C^{r}$ topology. Note that any two orthogonal embeddings of $S^{p}$ in $S^{q}$ are isotopic, since $p<q$. We define an action '.' of the direct product of the orthogonal groups $O(p+1) \times O(q+1)$ on $W$ by means of the identity

$$
((P, Q) \cdot g)(x)=Q g\left(P^{-1} x\right)
$$

where $(P, Q) \in O(p+1) \times O(q+1), g \in W, x \in S^{p}$.

Let $L$ be the fibre product of the principal bundles associated with $A, E$. Let $D$ be the bundle associated with $L$ and with fibre $W$. Then $f$ corresponds to a cross-section of $D$. The Stiefel manifold $V_{q+1, p+1}$ is the $O(p+1) \times O(q+1)$ invariant subspace $V$ of $W$ consisting of the orthogonal embeddings. Let $C$ be the bundle associated with $L$ and with fibre $V$. Then the inclusion $j$ of $V$ in $W$ extends to a fibre-preserving map from $C$ to $D$.

Taking derivatives at the basepoint of $S^{p}$ defines a retraction of $W$ onto $V$ and so $j_{*}: \pi_{k} V \rightarrow \pi_{k} W$ is the inclusion of a direct summand. By [4, Proposition 2] (see also [7]), $j_{*}$ is surjective when $k \leqslant 2 q-4 p-3$. We use this to prove the following lemma.

LEMMA 3. (i) If $\operatorname{dim} B \leqslant 2 q-4 p-2$ then there is a fibrewise orthogonal embedding of $A$ in $E$.

(ii) If $\operatorname{dim} B \leqslant 2 q-4 p-3$ then $f$ is isotopic to a fibrewise orthogonal embedding.

For the proof let $s$ be the cross-section of $D$ corresponding to $f$ and note that, since $j_{*}$ is an isomorphism when $k \leqslant 2 q-4 p-3$, the vertical homotopy class of $s \mid B^{2 q-4 p-3}$ comes from a cross-section $s_{1}$ of $C \mid B^{2 q-4 p-3}$. This proves (ii). To prove (i) let $\theta_{1} \in H^{2 q-4 p-2}\left(B ; \pi_{2 q-4 p-3} V\right)$ be the obstruction to extending $s_{1} \mid B^{2 q-4 p-4}$ to a cross-section of $C \mid B^{2 q-4 p-2}$. Then $j_{* *} \theta_{1} \in H^{2 q-4 p-2}\left(B ; \pi_{2 q-4 p-3} W\right)$ is the obstruction to extending $s \mid B^{2 q-4 p-4}: B^{2 q-4 p-4} \stackrel{s_{1}}{\rightarrow} C \rightarrow D$ to a cross-section of $D \mid B^{2 q-4 p-2}$. Since this obstruction is zero so also is $\theta_{1}$, and $s_{1} \mid B^{2 q-4 p-4}$ extends to a cross-section of $C \mid B^{2 q-4 p-2}$. But cross-sections of $C$ correspond bijectively and 
naturally to fibrewise orthogonal embeddings of $A$ in $E$. This proves Lemma 3.

3. The general case. For the applications in $\$ 4$ we take $p=1$, and the most interesting values of $q$ are probably $2,3,4$. When $p=1$ Lemma 3(i) holds trivially for these values of $q$, without the hypothesis that there is a fibrewise $C^{r}$ embedding of $A$ in $E$. We therefore need a more general result.

We continue to work in the context of $\S 2$ and include $E$ in its fibre suspension $\Sigma E$ in the usual way. Following $f$ by $j$ such inclusions $(j \geqslant 1)$ we obtain a fibrewise $C^{r}$ embedding $f^{\prime}$ of $A$ in $E^{\prime}=\Sigma^{j} E$.

LEMMA 1. The inclusion of $\Sigma^{j}(E-f(A))$ in $E^{\prime}-f^{\prime}(A)$ is a fibre homotopy equivalence.

By $\S 2$, Lemma 1 both $\Sigma^{j}(E-f(A))$ and $E^{\prime}-f^{\prime}(A)$ are fibre bundles. Therefore, by a result due to Dold [1], it suffices to prove that the inclusion is a homotopy equivalence over the basepoint. But the inclusion $S(X-Y) \rightarrow S X-Y$ is a homotopy equivalence whenever $(X, Y)$ is a polyhedral pair with $X, Y$ compact. Iterating this $j$ times with $X=M, Y=F$ we have Lemma 1.

LEMMA 2. Let $S^{p}$ be embedded orthogonally in $S^{q}$ and let $S^{q-p-1}$ be the $q-p-1$-sphere orthogonal to $S^{p}$. Then the inclusion of $S^{q-p-1}$ in $S^{q}-S^{p}$ is a homotopy equivalence.

One proof is by induction on $q$, beginning at $q=p+1$ and arguing as in the proof of Lemma 1 .

Lemma 3. Suppose that $j \geqslant[(\operatorname{dim} B+1) / 2]+2 p-q+2$. Then $E^{\prime}=\Sigma^{j} E$ is the fibre join of $A$ with a bundle $E^{\prime \prime}$ which is fibre homotopy equivalent to the $j$-fold fibre suspension of a homotopy $q-p-1$-sphere bundle.

To prove the lemma we apply $\$ 2$, Lemma 3(ii) to the fibrewise $C^{r}$ embedding $f^{\prime}$ of $A$ in $E^{\prime}$. Thus $f^{\prime}$ is isotopic to a fibrewise orthogonal embedding $f^{\prime \prime}$. Let $E^{\prime \prime}$ be the orthogonal complement to $f^{\prime \prime}(A)$ in $E^{\prime}$. Then $E^{\prime}$ is equivalent to the fibre join of $A$ with $E^{\prime \prime}$. But by Lemma 2 the inclusion of $E^{\prime \prime}$ in $E^{\prime}-f^{\prime \prime}(A)$ is a fibre homotopy equivalence. But $E^{\prime}-f^{\prime \prime}(A)$ is equivalent to $E^{\prime}-f^{\prime}(A)$ by $\S 2$, Lemma 2 , and $E^{\prime}-f^{\prime}(A)$ is fibre homotopy equivalent to $\Sigma^{j}(E-f(A))$ by Lemma 1 . But $E^{\prime}-f(A)$ is a homotopy $q-p-1$-sphere bundle by Lemma 2 . This completes the proof.

When $q=p+1$ we also have the following result.

Lemma 4. Let $q=p+1$. Then $E$ is the fibre join of $a$ 0-sphere bundle with an orthogonal $p$-sphere bundle $A^{*}$ which is fibre homotopy equivalent to $A$.

To prove this note, by $\S 2$, Lemma 1 , the complement $E-f(A)$ is a $C^{r}$ bundle over $B$ whose fibre is the disjoint union of two copies of an open $q$-disc. Since the $q$-disc is contractible we may continuously assign to each $b \in B$ a subset $\left\{y_{1}, y_{2}\right\}$ of $E_{b}-f\left(A_{b}\right)$ so that $y_{1}, y_{2}$ lie in different path components. Let $A_{b}^{*}$ be the $p$-sphere orthogonal to a geodesic joining $y_{1}, y_{2}$. Let $E_{b}^{*}$ be the 0-sphere orthogonal to $A_{b}^{*}$ in $E_{b}$. Then the $A_{b}^{*}, E_{b}^{*}$ make up an orthogonal $p$-sphere bundle $A^{*}$ and a 0 -sphere 
bundle $E^{*}$. But $E$ is the fibre join of $A^{*}$ with $E^{*}$. To complete the proof we note that both $A^{*}$ and $A$ are fibre homotopy equivalent to the bundle $E-$ $\cup_{b \in B}\left\{y_{1}, y_{2}\right\}$.

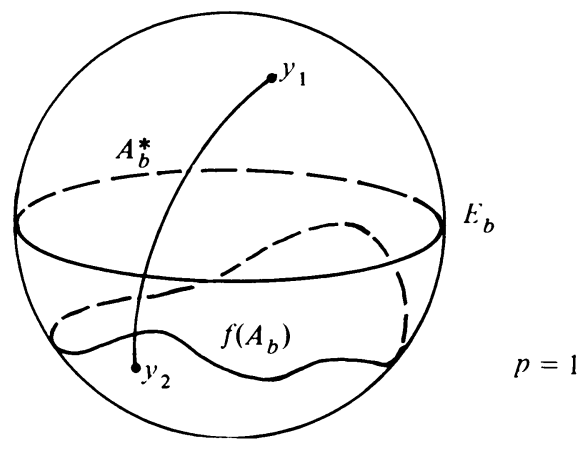

4. Applications. We resume the discussion of $\S 1$. For $q \geqslant 1$ let $E$ be an orthogonal $q$-sphere bundle over a connected finite CW-complex $B$. Let $A_{0}=F$ be a circle embedded orthogonally in $M=S^{q}$. If $K$ is empty then the attractors $A_{b}$ make up a circle bundle $A^{\prime}$ over $B$. From the definition of an attractor each $V_{b} \mid A_{b}$ has at most one zero. Therefore, and since all points are regular, $A^{\prime}$ is oriented. If $q=1$ this means that $E$ is orientable. Evidently the converse also holds. If $q=1$ and $E$ is orientable then $K$ can be empty. From now on we suppose that $q>2$.

If $K$ is empty and $V_{0} \mid A_{0}$ has a zero then, by regularity, so has each other $V_{b} \mid A_{b}$ and the zeros define a cross-section of $A^{\prime}$. Therefore $A^{\prime}$ is trivial, and so $E$ has two orthogonal cross-sections. It follows that the trivial circle bundle $A^{\prime}$ embeds fibrewise orthogonally in $E$.

If $K$ is empty and $V_{0} \mid A_{0}$ is never zero then, by regularity, so is each other $V_{b} \mid A_{b}$. Therefore the flow $\phi_{V_{b}}$ defines a $C^{r}$ embedding of $S^{1}$ in $E_{b}$ whose image is $A_{b}$ and whose derivative maps the clockwise unit tangent field on $S^{1}$ to $V_{b} \mid A_{b}$. Such embeddings are unique up to rotations of $S^{1}$. Therefore $A^{\prime}$ is the image by a fibrewise $C^{r}$ embedding of an oriented orthogonal circle bundle $A$. Summarizing, we have the first part of the following lemma.

LEMMA 1. (i) If $K$ can be empty then there is a fibrewise $C^{r}$ embedding of an oriented orthogonal bundle $A$ in $E$.

(ii) The converse also holds. We prove this in an appendix.

Comparing Lemma 1(i) with §3, Lemma 4 we have the following result.

TheOREM 1. Let $q=2$. If $K$ can be empty then $E$ is the fibre join of an oriented orthogonal circle bundle with a 0 -sphere bundle. (Of course the converse is contained in Lemma 1(ii).)

The next result is a consequence of Lemma 1(i) and §2, Lemma 3(i).

THEOREM 2. Suppose that $\operatorname{dim} B \leqslant 2 q-6$. If $K$ can be empty then $E$ is the fibre join of an oriented orthogonal circle bundle with an orthogonal $q-2$-sphere bundle. (Of course the converse is contained in Lemma 1(ii).) 
Corollary. Let $E$ be orientable.

(i) If $q \geqslant 6$ and if $K$ can be empty then for some $a \in H^{2}(B ; Z)$ the sum

$$
W_{q}+a W_{q-2}+a^{2} W_{q-4}+\cdots
$$

is zero.

(ii) If $\operatorname{dim} B \leqslant q$ and if for some a the sum in (i) is zero then $K$ can be empty.

Here $W_{i}$ is the $i$ th Stiefel-Whitney class of $E$ and we work in $H^{*} B$ with coefficients $\mathbf{Z}$ or $Z / 2 Z$ accordingly as $q$ is odd or even. To prove (i) we apply the Whitney duality theorem with Theorem 2. To prove (ii) we apply [6, Proposition 2.1] together with Lemma 1(ii).

Theorem 3. Let $q=3,4,5$ and let $j=[(\operatorname{dim} B+1) / 2]-q+4$. If $K$ can be empty then $\Sigma^{j} E$ is the fibre join of an oriented orthogonal circle bundle with a bundle which is fibre homotopy equivalent to the j-fold fibre suspension of a homotopy $q$ - 2-sphere bundle. (Of course a converse is contained in Lemma 1(ii).)

To prove Theorem 3 we compare Lemma 1(i) with §3, Lemma 3.

Corollary. Let $q=3,4,5$. If $K$ can be empty then for some $a \in H^{2}(B ; Z)$ the mod 2 reduction of the sum in the first part of the corollary to Theorem 2 is zero. (Of course a converse is contained in the second part of the corollary to Theorem 2.)

To prove this we apply the mod 2 Whitney duality theorem with Theorem 3.

Examples. (i) If $B$ is a sphere then $K$ can be empty if and only if $E$ has two orthogonal cross-sections. This follows from Lemma 1 together with the remark in $\S 2$.

(ii) Let $B$ be a closed oriented 4-manifold such that for all $a \in H^{2}(B ; Z)$ the mod 2 reduction of $a^{2}$ is zero. For instance $S^{2} \times S^{2}$ would do. Let $g: B \rightarrow S^{4}$ be a degree 1 map. Let $H$ be the Hopf 3-sphere bundle $S^{7}$ over $S^{4}$. Then let $E$ be the pullback $g^{*} \Sigma H$ of the fibre suspension $\Sigma H$. We have $W_{0}=1, W_{1}=W_{2}=W_{3}=0$ and $W_{4} \neq 0$. Therefore by the corollary to Theorem 3 the catastrophe space $K$ must be nonempty.

There remains only an appendix for the proof of Lemma 1(ii). I wish to thank Dr. A. du Plessis and Professor M. G. Barratt for their helpful comments. I am especially grateful to Professor Barratt for his hospitality during the winter of 1976-77.

Appendix. Let $E$ be a $C^{r}$ bundle with fibre a closed $C^{r}$ manifold $M(r \geqslant 1)$ over a connected finite CW-complex $B$. Let $A^{\prime}$ be an oriented orthogonal circle bundle over $B$. The purpose of this appendix is to prove the following result.

Proposition. If there is a fibrewise $C^{r}$ embedding of $A^{\prime}$ in $E$ then $K$ can be empty with $A_{0}=A_{0}^{\prime}$.

The proof is modelled on that of the corresponding result in $\S 1$ for point attractors. As in $\$ 1$ we form the vector bundles $T F E, T F A^{\prime}$ over $E, A^{\prime}$. We identify $A^{\prime}$ with the image in $E$. Then the line bundle $T F A^{\prime}$ is a subbundle of $T F E \mid A^{\prime}$. We choose a Riemannian metric on $T F E \mid A^{\prime}$. 
If $\operatorname{dim} M=1$ then $M$ is a disjoint union of circles and the proposition holds trivially. If $\operatorname{dim} M>1$ we define $N F A^{\prime}$ to be the orthogonal complement to $T F A^{\prime}$ in $T F E \mid A^{\prime}$. Then $N F A^{\prime}$ is a Riemannian vector bundle over $A^{\prime}$. Using the compactness of $B$ we choose $\delta>0$ so that the fibrewise exponential map $\varepsilon$ : $N F A^{\prime} \rightarrow E$ maps the open disc bundle $B_{\delta}$ of radius $\delta$ homeomorphically into $E$. Then $N=\varepsilon\left(B_{\delta}\right)$ is a neighbourhood of $A^{\prime}$ in $E$.

We identify $N F A^{\prime}$ with its own fibrewise tangent bundle and define a family $R_{b}$ $(b \in B)$ of fibrewise $C^{r-1}$ vector fields on $N$ by means of the identity $R_{b}(e)=$ $-d \varepsilon_{b}(t)$ where $e \in E_{b}$ and $e=\varepsilon(t)$ for $t \in B_{\delta}$. The orientation defines a family of fibrewise $C^{r-1}$ unit vector fields on $A^{\prime}$. We extend this to a family $C_{b}(b \in B)$ of fibrewise $C^{r-1}$ vector fields on $N$. Then for $e \in E_{b}$ we define $V_{b}(e)$ to be either 0 or

$$
C_{b}(e)+R_{b}(e) \exp (-\sec \theta)
$$

accordingly as $e \notin N$ or $e=\varepsilon(t) \in N$ for $t \in B_{\delta}$. Here $\theta=\pi\|t\| / 2 \delta$. Taking $A_{b}=A_{b}^{\prime}$ we see that $K$ is empty. This completes the proof.

\section{REFERENCES}

1. A. Dold, Über fasernweise Homotopieaquivälenz von Faserräumen, Math. Z. 62 (1955), 111-136.

2. E. L. Lima, On the local triviality of the restriction map for embeddings, Comment. Math. Helv. 38 (1964), 163-164.

3. N. E. Steenrod, The topology of fibre bundles, Princeton Univ. Press, Princeton, N. J., 1951.

4. A. S. Svarc, Homology of spaces of smooth embeddings, Soviet Math. Dokl. 1 (1966), 339-342.

5. R. Thom, Structural stability and morphogenesis, Benjamin, New York, 1975.

6. P. E. Thomas, Fields of tangent $k$-planes on manifolds, Invent. Math. 3 (1967), 334-347.

7. A. M. Vinogradov, Some homotopic properties of the space of embeddings of a circle into a sphere or ball, Soviet Math. Dokl. 5 (1964), 737-740.

Department of Mathematics, Untversity of Western australia, Nedlands, Western Auștralia 6009, Australia

Current address: Department of Mathematics, Massachusetts Institute of Technology, Cambridge, Massachusetts 02139 